\title{
Delivering the promise of RNA therapeutics
}

\author{
As the clinical potential of RNA therapeutics begins to be unveiled, expanding the range of tissue types that can be \\ targeted for delivery of these drugs is now the main hurdle to overcome.
}

T

he concept of RNA-targeting therapeutics-using antisense oligonucleotides (ASOs), aptamers, siRNAs, miRNAs and, more recently, synthetic mRNAs-to control the expression of disease-relevant genes as a way to treat illness is an alluring one. Such drugs have the ability to engage targets that are otherwise 'undruggable' by small molecules and proteins, thus opening up whole new avenues for treating intractable diseases. Turning this concept into a clinical reality, however, is no small feat. ASOs and siRNAs have been in clinical development for decades, and for much of this time, clinical success has been out of reach. New medicinal chemistry needed to be invented to make the molecules more potent and less immunogenic, and the delivery hurdlegetting the drug into the right cell of the right tissue- has been a major roadblock. Now, after years of slow progress, the field is picking up steam. In 2016, nusinersen, a splicing switching ASO, was approved by the FDA and became the first drug to treat spinal muscular atrophy, a rare and often fatal disease of the nervous system, and 2018 witnessed the first ever approval of an RNAi drug - patisiran - to treat hereditary transthyretin amyloidosis (hATTR), another rare and devastating disease mediated by the liver. These recent successes validated the clinical utility of RNA-targeting therapeutics, and brought forward lifesaving drugs for patients who previously had no effective treatment options.

One enticing feature of RNA-targeting therapeutics is that once chemistry and delivery are optimized, designing and producing a lead compound for a new target is relatively straightforward, and their in vivo pharmacokinetics are highly predictable. This means that the timeline from target identification to preclinical proof of concept in animal models, to having a lead compound ready to be tested in clinical trials, can be as short as 6 months-some would estimate-rather than years, which has been the norm for drug development until now. This is reflected in a burgeoning clinical pipeline: currently more than a hundred investigational RNA-targeting drugs are under clinical development for disease indications encompassing neurodegeneration, metabolic and cardiovascular disorders and various cancers.

For good reason, as of now most of the RNA-targeting drug candidates in clinical trials are directed at the liver. A major breakthrough in the field was the discovery that conjugation of GalNac, an amino sugar moiety, to siRNA and ASO drugs can lead to efficient liver targeting and cellular uptake due to the unusually high expression of its receptor on the surface of hepatocytes. One interesting example is inclisiran, a GalNacconjugated siRNA drug that knocks down the expression of PCSK9 to reduce LDL cholesterol. In a previous phase 2 trial, a single subcutaneous injection of inclisiran resulted in about $50 \%$ reduction of PCSK9 level 6 months after the initial dosing (Ray, K. et al., N. Engl. J. Med. 376, 1430-1440; 2017). The drug is now being evaluated in multiple pivotal trials to treat patients with cardiovascular disease and elevated LDL. If successful, it will serve as a prime example where infrequent and convenient dosing, coupled with a good safety profile, might enable RNA-targeting therapeutics to go beyond rare diseases and enter the realm of common diseases, which affect a large proportion of the population.

Beyond liver-mediated diseases, RNAtargeting therapeutics is also likely to have a transformative impact in the treatment of genetic diseases of the central nervous system-an area of large, unmet medical need. There, the challenge of getting the drug into the right tissue can be partially overcome through local delivery such as intrathecal injection. Building on the success of nusinersen, two other CNStargeting ASOs are currently in phase 3 trials. IONIS-HTTR $\mathrm{x}$ was designed to lower the mutated huntingtin protein, which causes Huntington disease, and tofersen to reduce the SOD1 protein that leads to a familial form of amyotrophic lateral sclerosis. If successful, they may become the first disease-modifying drugs for these two diseases, which currently are universally lethal. Such success may also lay out a path for tackling other CNS disorders with more complex genetic makeup, such as Alzheimer's disease.

In the long run, the development of innovative delivery technologies that enable safe and efficient targeting of additional tissues will be the key to expanding RNAtargeting therapeutics to a broad range of clinical indications. To that regard, progress is happening in the preclinical front. For example, nanoparticles are being used to deliver RNA-targeting drugs, and their formulation has been tweaked to enable delivery of the payload to non-liver tissues, such as the lung (Dahlman, J. et al., Nature Nanotechnol. 9, 648-655; 2014). Conjugation of siRNAs with antibodies that are capable of recognizing specific surface receptors can direct the drugs to muscle tissue (Sugo, T. et al., J. Control. Release 237, 1-13; 2016) and to certain cancer cells (Bäumer, S. et al., Clin. Cancer Res. 21, 1383-1394; 2015). Exosomes also have been explored as an alternative to synthetic nanoparticles, and have been successfully used to deliver siRNAs against KRAS in a mouse model of pancreatic cancer (Kamerkar, S. et al., Nature 546, 498-503; 2017). Whether these and the other ongoing approaches can be successfully translated into the clinic remains to be seen.

The successes of ASOs and siRNAs, together with the potential of miRNAs and mRNAs, suggest that we are witnessing the dawn of RNA-targeting therapeutics. It is now time for the community to focus on developing clinically viable delivery approaches that take these drugs into a wider range of tissues. If and when the delivery challenges are solved, many breakthrough treatments for patients will be within reach.

Published online: 9 September 2019 https://doi.org/10.1038/s41591-019-0580-6 